\title{
Synthesis and biological activity of Citridone $A$ and its derivatives
}

\author{
Takashi Fukuda, Kenta Shimoyama, Tohru Nagamitsu and Hiroshi Tomoda \\ Citridone A (1), originally isolated as a potentiator of antifungal miconazole activity from a fungal culture broth, has a \\ phenyl- $R$-furopyridone structure. Because of its unique ring structure, 11 derivatives were chemically synthesized and their \\ biological activity was evaluated. Derivatives 17,20 and 21 potentiated miconazole activity against Candida albicans. \\ Furthermore, 1, 14, 20 and 21 were found to inhibit yellow pigment production in methicillin-resistant Staphylococcus aureus. \\ The Journal of Antibiotics (2014) 67, 445-450; doi:10.1038/ja.2014.14; published online 19 March 2014
}

Keywords: 3-phenyl-4-hydroxy-2-pyridone; fungal metabolite; miconazole potentiator; MRSA; staphyloxanthin inhibitor

\section{INTRODUCTION}

Citridone A (1) (Figure 1), a potentiator of miconazole activity against Candida albicans, was originally isolated from the culture broth of Penicillium sp. FKI-1938..$^{-3}$ Compound 1 has a rare phenyl$R$-furopyridone skeleton (6-6/5/5 ring system) and 1 was the only natural compound having this ring system. Structurally related citridones 2-4 (Figure 1) were also isolated from the fungus, but they showed very weak miconazole-potentiating activity. ${ }^{3}$ Because of its unique structure and biological properties, two groups have already completed its total synthesis. ${ }^{4,5}$ These findings prompted us to synthesize new citridone A derivatives to understand the structureactivity relationship. Furthermore, the biological activity of the derivatives was re-evaluated in 15 in-house assay systems. Among 11 derivatives synthesized in this study (Figure 1), 3 derivatives, 17, 20 and 21, potentiated miconazole activity against C. albicans. Interestingly, 1, 14, 20 and 21 were found to inhibit yellow pigment production in methicillin-resistant Staphylococcus aureus (MRSA).

In this study, we described the synthesis of citridone derivatives and their biological activity, including the potentiating activity of antifungal miconazole and inhibitory activity of yellow pigment production in MRSA.

\section{RESULTS AND DISCUSSION}

Citridone A (1) and its 11 derivatives $(7,11,14,15,16,17,19,20,21$, 27 and 29) were synthesized. ${ }^{4}$ Derivatives 11, 17 and 21 (enantiomer of 1) were prepared as shown in Chart 1 , according to the total synthesis of $\mathbf{1}$ we achieved previously. ${ }^{4}$ Intermediate 5 was exposed to tetra- $n$-butylammonium fluoride to afford 7 , a new derivative without a dihydrofuran ring. The reactions of other intermediates 11 and 12 with $t$-BuOK proceeded via cyclobutane formation, followed by novel pyrolysis ${ }^{6-8}$ to give alkenes 14 and 15 . Deprotection of 15 and reduction of aldehyde 17 gave the new derivatives 16 and 19, respectively. In addition, 10, which was synthesized by regioselective iodocyclization according to our established method, ${ }^{4}$ was converted to regioisomer 20 (Chart 1). Derivatives 27 and 29 were also prepared as shown in Chart 2. The $\operatorname{Pd}(0)$-catalyzed coupling reaction between 22 and $23^{4}$ followed by heating at $210^{\circ} \mathrm{C}$ afforded pyridone 25 . Regioselective iodocyclization under different conditions produced the corresponding iodides 26 and 28, from which E2 elimination then gave the desired derivatives 27 and 29 , respectively.

Potentiation of miconazole activity against C. albicans in combination with citridones and their derivatives (Figure 1) was assayed by the conventional method using paper disks. ${ }^{1}$ None of the citridones themselves showed any inhibition against C. albicans at up to $20 \mu \mathrm{g}$ per disk on plate A (without miconazole). Citridone A (1) and derivatives 17, 20 and 21 ( $20 \mu \mathrm{g}$ per $6 \mathrm{~mm}$ disk) were found to potentiate miconazole activity by forming inhibitory zones around the paper disks on plate $\mathrm{B}$ in contact with a small amount of miconazole $(60 \mathrm{~nm}$, which, at this level, had no effect on the growth of C. albicans; $21,15,16,21 \mathrm{~mm})$; however, the other compounds $(20 \mu \mathrm{g}$ per $6 \mathrm{~mm}$ disk) showed no potentiation activity. Citridone A and its enantiomer (21) showed the largest inhibition zone on plate B (Table 1).

As the structures of citridone derivatives are unique as small molecules, other biological activities of the derivatives were evaluated. Inhibitory activity of yellow pigment production in MRSA was assayed by our established method using paper disks. ${ }^{9}$ Compounds 1, 14, 20 and 21 ( $20 \mu \mathrm{g}$ per $6 \mathrm{~mm}$ disk) were found to inhibit yellow pigment production by forming white zones around the paper disks $(16,13,15,15 \mathrm{~mm})$; however, the other compounds (20 $\mu \mathrm{g}$ per $6 \mathrm{~mm}$ disk) showed no activity. Similar results were obtained when using methicillin-sensitive $S$. aureus (MSSA) instead of MRSA (Table 1). 


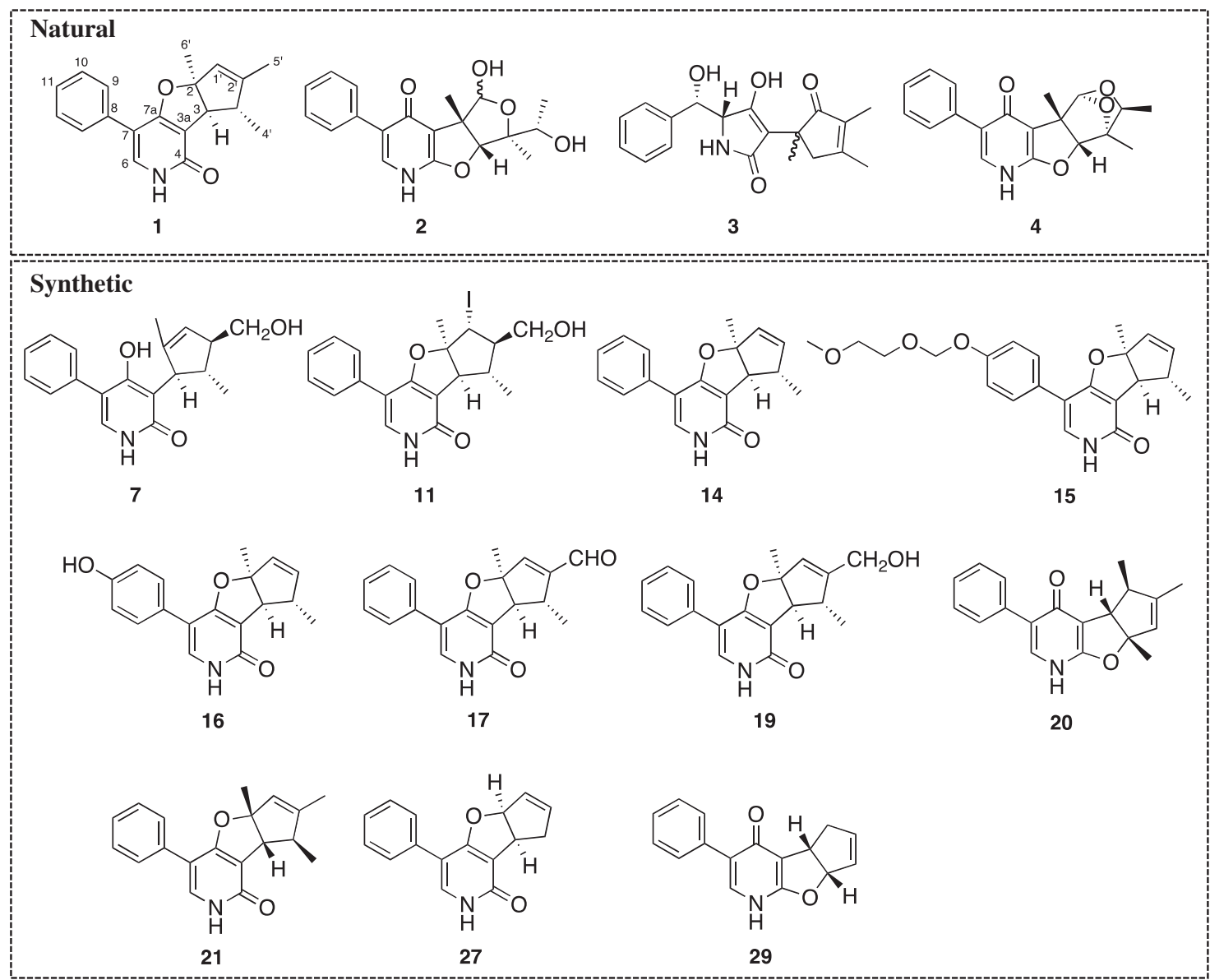

Figure 1 Structures of natural and synthetic citridones.

To confirm the inhibition of MRSA yellow pigment production by these compounds, they were evaluated by the liquid culture method. ${ }^{9}$ As summarized in Table 1, compounds 1, 14, 20 and 21 inhibited yellow pigment production with $\mathrm{IC}_{50}$ values of $11.1 \mu \mathrm{g} \mathrm{ml}^{-1}$, $30.0 \mu \mathrm{g} \mathrm{ml}^{-1}, 22.5 \mu \mathrm{g} \mathrm{ml}^{-1}$ and $22.7 \mu \mathrm{g} \mathrm{ml}^{-1}$, respectively, without any effect on the growth of MRSA at $30 \mu \mathrm{g} \mathrm{ml}^{-1}$.

It is well known that $S$. aureus produces a yellow pigment called staphyloxanthin. ${ }^{10,11}$ Recently, several research groups reported that staphyloxanthin is one of the important virulent factors of S. aureus. ${ }^{12,13}$ Staphyloxanthin acts as an antioxidant with its numerous conjugated double bonds, which enable $S$. aureus to survive by detoxification of host-generated reactive oxgen species such as $\mathrm{O}_{2}{ }^{-}, \mathrm{H}_{2} \mathrm{O}_{2}$ and $\mathrm{HOCl}{ }^{14,15}$ Staphyloxanthin develops in the cell membrane of $S$. aureus and is associated with enhancing $S$. aureus survival and infection. ${ }^{16}$

Staphyloxanthin is composed of a glucose core to which a prenyl residue and a fatty acyl residue are attached. The biosynthetic pathway of staphyloxanthin was reported previously. ${ }^{11}$ Importantly, a CrtMdeficient mutant, which lacks an enzyme involved in synthesis of the prenyl residue and cannot produce staphyloxanthin, was reported to fail to survive in the host mouse. ${ }^{17,18}$ Recently, several squalene synthase inhibitors, BPH- $652,{ }^{19}$ zaragozic acid, ${ }^{20}$ rhodomyrtone ${ }^{21}$ and tylopilusins, ${ }^{22}$ were found to inhibit staphyloxanthin production in $S$ aureus. Furthermore, BPH-652 was demonstrated to block infection of the host mouse with $S$. aureus. ${ }^{19}$ Therefore, the staphyloxanthin biosynthetic pathway of $S$ aureus is expected to offer a new potential target to combat MSSA and MRSA infection.
Citridone A and derivatives 20 and 21, having a common 4,5,6a-trimethyl-4,6a-dihydro-3aH-cyclopentafuran skeleton, showed both biological activities. It is very difficult to imagine that both activities are derived from the same mechanism of action. Further precise analysis is necessary to demonstrate the mechanisms.

\section{MATERIALS AND METHODS}

\section{General experimental procedures}

Ultraviolet spectra were recorded on a spectrophotometer (8453 UV-Visible spectrophotometer; Agilent Technologies Inc., Santa Clara, CA, USA). Optical rotations were measured with a digital polarimeter (DIP-1000; JASCO, Tokyo, Japan). HR-ESI-TOF-mass spectra were recorded on a mass spectrometer (JMS-T100LP; JEOL, Tokyo, Japan). Various NMR spectra were measured with a spectrometer (XL-400; Varian, Inc., Palo Alto, CA, USA).

\section{Experimental procedures and characterization data}

Stocked natural citridones B to D (2-4) used for this investigation were isolated from a culture broth of Penicillium sp. FKI-1938. ${ }^{1,3}$

All experimental procedures for the synthesis of compounds $(7,11,14,15$, $16,17,19,20,21,27$ and 29), including citridone A (1), are summarized in Supplementary Information.

(5aS,8R,8aS)-5a,7,8-Trimethyl-4-phenyl-2,5a,8,8a-tetrahydro-1H-cyclopenta [4,5] furo[3,2-c]pyridin-1-one (citridone $A, 1) . \quad[\alpha]_{\mathrm{D}}^{21}-76.9\left(c 1.0, \mathrm{CH}_{3} \mathrm{OH}\right)$. IR $(\mathrm{KBr}) \mathrm{cm}^{-1}: 2926,1653,1431,1231,1030,763,697 .{ }^{1} \mathrm{H}-\mathrm{NMR}(300 \mathrm{MHz}$, $\left.\mathrm{CDCl}_{3}\right) \delta: 7.55-7.51(\mathrm{~m}, 2 \mathrm{H}), 7.45(\mathrm{~s}, 1 \mathrm{H}), 7.42-7.36(\mathrm{~m}, 2 \mathrm{H}), 7.33-7.27$ $(\mathrm{m}, 1 \mathrm{H}), 5.39(\mathrm{dq}, J=1.7 \mathrm{~Hz}, 1 \mathrm{H}), 3.27(\mathrm{~d}, J=1.7 \mathrm{~Hz}, 1 \mathrm{H}), 2.91$ (bq, $J=7.2$ 


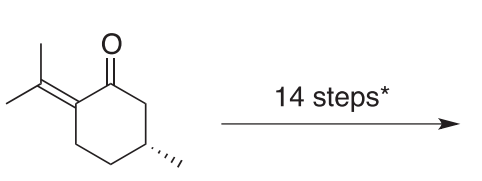<smiles>[R]c1ccc(-c2c[nH]c(=O)c(C3C(C)=CC(CO[SbH2])C3C)c2O)cc1</smiles>

$5^{*}(\mathrm{R}=\mathrm{H})$

$6(\mathrm{R}=\mathrm{OMEM})$<smiles>CC1=C[C@@H](CO)[C@H](C)[C@H]1c1c(O)c(-c2ccccc2)c[nH]c1=O</smiles>

7

(+)-pulegone

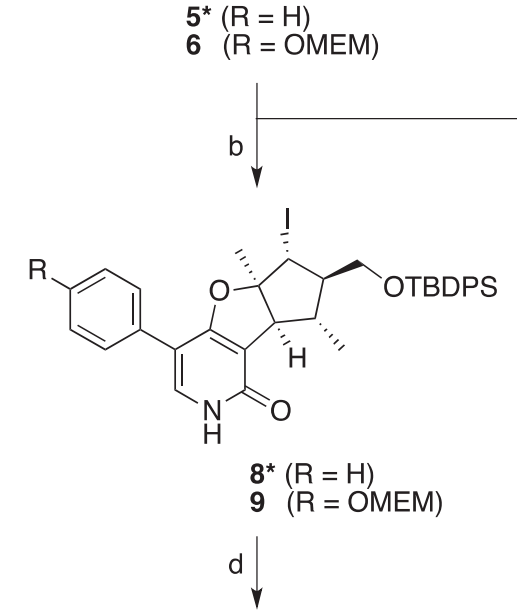

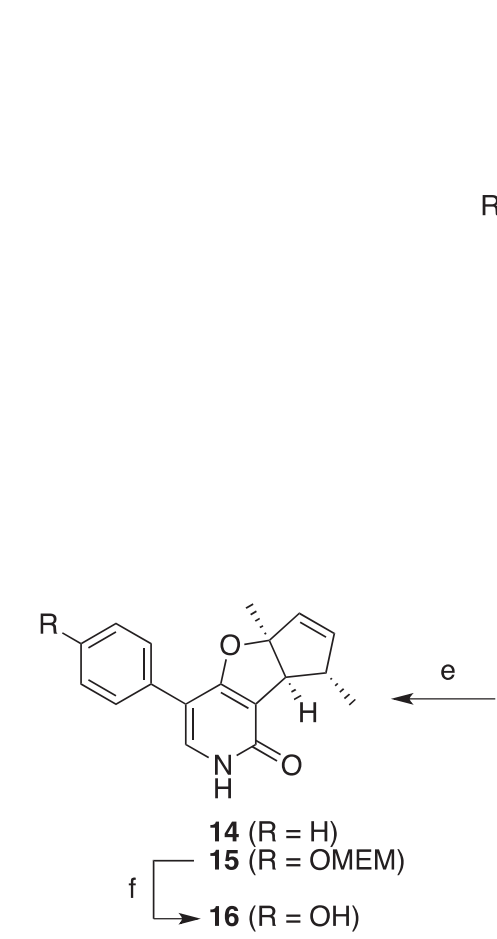<smiles>C[C@H]1C(CO)=C[C@]2(C)Oc3c(-c4ccccc4)c[nH]c(=O)c3[C@H]12</smiles>

19

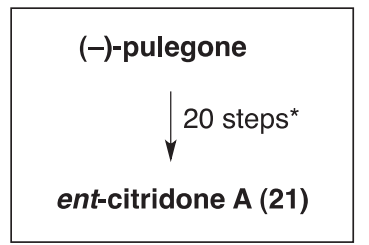<smiles>[R]c1ccc(-c2c[nH]c(=O)c3c2O[C@@]2(C)[C@H]3[C@H](C)[C@H](CO)[C@@H]2I)cc1</smiles>

$11^{*}(\mathrm{R}=\mathrm{H})$

$12(\mathrm{R}=\mathrm{OMEM})$

g,h<smiles>C[C@H]1C(C=O)=C[C@]2(C)Oc3c(-c4ccccc4)c[nH]c(=O)c3[C@H]12</smiles>

$17^{*}$

$\mathrm{j}, \mathrm{k}$<smiles>CC1=C[C@]2(C)Oc3c(-c4ccccc4)c[nH]c(=O)c3[C@H]2[C@H]1C</smiles>

citridone $A(1)^{*}$

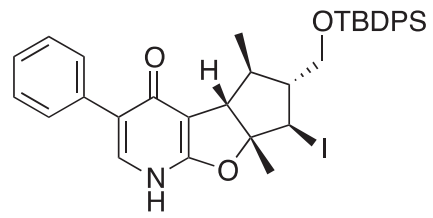

$10^{*}$

$\downarrow d$<smiles>CC1[C@H]2c3c([nH]cc(-c4ccccc4)c3=O)O[C@@]2(C)[C@H](I)[C@H]1CO</smiles>

13

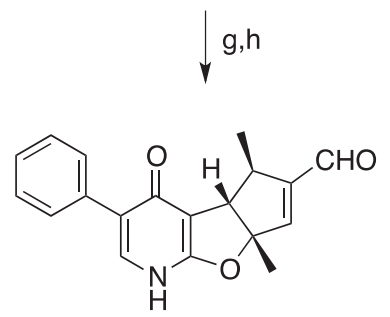

18

$j, k$<smiles>CC1=C[C@]2(C)Oc3[nH]cc(-c4ccccc4)c(=O)c3[C@H]2[C@H]1C</smiles>

20

Chart 1 Preparation of compounds 7, 11,14,15,16,17, 19, 20 and 21. Reagents and conditions: (a) tetra- $n$-butylammonium fluoride, tetrahydrofuran, room temperature, 88\%; (b) $\mathrm{N}$-iodosuccinimide, pyridine, $\mathrm{CH}_{2} \mathrm{Cl}_{2}$, room temperature*; (c) $\mathrm{I}_{2}, \mathrm{CH}_{2} \mathrm{Cl}_{2}$, room temperature*; (d) tetra- $n$-butylammonium fluoride, tetrahydrofuran, room temperature, $88 \%$ for $11^{*}, 89 \%$ for $12,77 \%$ for 13 ; (e) $t$-BuOK, $N, N$-dimethylformamide. $100{ }^{\circ} \mathrm{C}, 88 \%$ for 14 , $77 \%$ for 15; (f) trifluoroacetic acid, $\mathrm{CH}_{2} \mathrm{Cl}_{2}, 0{ }^{\circ} \mathrm{C}, 92 \%$; (g) Dess-Martin Periodinane, $\mathrm{CH}_{2} \mathrm{Cl}_{2}$, dimethyl sulfoxide, pyridine, room temperature; (h) 1,8-diazabicyclo[5.4.0] undec-7-ene, $\mathrm{CH}_{2} \mathrm{Cl}_{2}$, room temperature, $99 \%$ (2 steps) for $17^{*}, 65 \%$ (2 steps) for 18; (i) $\mathrm{NaBH}_{4}, \mathrm{CeCl}_{3} \cdot 7 \mathrm{H}_{2} \mathrm{O}$, $\mathrm{MeOH},-78^{\circ} \mathrm{C}, 77 \%$; (j) $\left(\mathrm{TMSSCH}_{2}\right)_{2}, \mathrm{ZnI}_{2}, \mathrm{CH}_{2} \mathrm{Cl}_{2}, \mathrm{Et}_{2} \mathrm{O}$; (k) $\mathrm{Bu}_{3} \mathrm{SnH}$, azobisisobutyronitrile, benzene, $55 \%$ (2 steps) for $\mathbf{1}^{*}, 40 \%$ (2 steps) for 20. *See, ref. 1. 


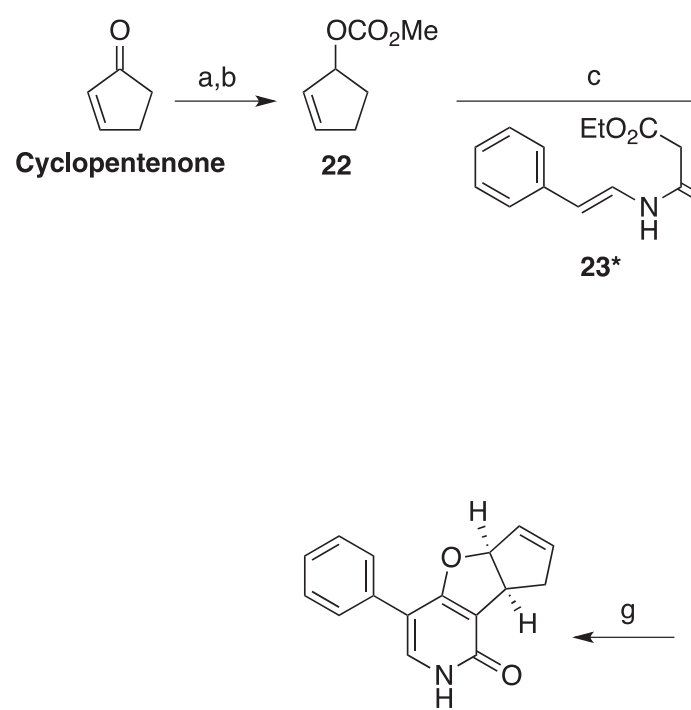

(士)-27

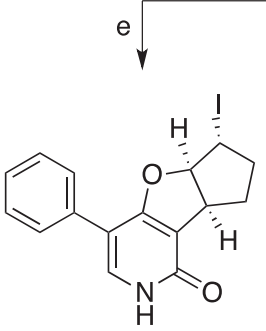

( \pm -26<smiles>CCOC(C(=O)N/C=C/c1ccccc1)C1C=CCC1</smiles>

24<smiles>O=c1[nH]cc(-c2ccccc2)c(O)c1C1C=CCC1</smiles>

25

Chart 2 Preparation of compounds 27 and 29. Reagents and conditions: (a) diisobutylaluminium hydride, $\mathrm{CH}_{2} \mathrm{Cl}_{2},-78{ }^{\circ} \mathrm{C} ;(\mathrm{b}) \mathrm{ClCO}_{2} \mathrm{Me}, \mathrm{N}, \mathrm{N}, \mathrm{N}^{\prime}, \mathrm{N}^{\prime}-$ tetramethylethylenediamine, $\mathrm{CH}_{2} \mathrm{Cl}_{2}, 0{ }^{\circ} \mathrm{C}, 92 \%$ (2 steps); (c) (allylPdCl) $2,1,1^{\prime}$-bis(diphenylphosphino)ferrocene, $\mathrm{NaH}$, tetrahydrofuran, room temperature, quant.; (d) $\mathrm{Ph}_{2} \mathrm{O}, 210^{\circ} \mathrm{C}, 86 \%$; (e) $\mathrm{N}$-iodosuccinimide, pyridine, $\mathrm{CH}_{2} \mathrm{Cl}_{2}$, room temperature, $61 \%$; (f) $\mathrm{I}_{2}, \mathrm{CH}_{2} \mathrm{Cl}_{2}$, room temperature, $83 \%$; (g) $t$ - $\mathrm{BuOK}$, $\mathrm{N}, \mathrm{N}$-dimethylformamide, $100{ }^{\circ} \mathrm{C}, 95 \%$ for $( \pm)-27,93 \%$ for $( \pm)-29$.

\section{Table 1 Summary of biological activity of citridone derivatives}

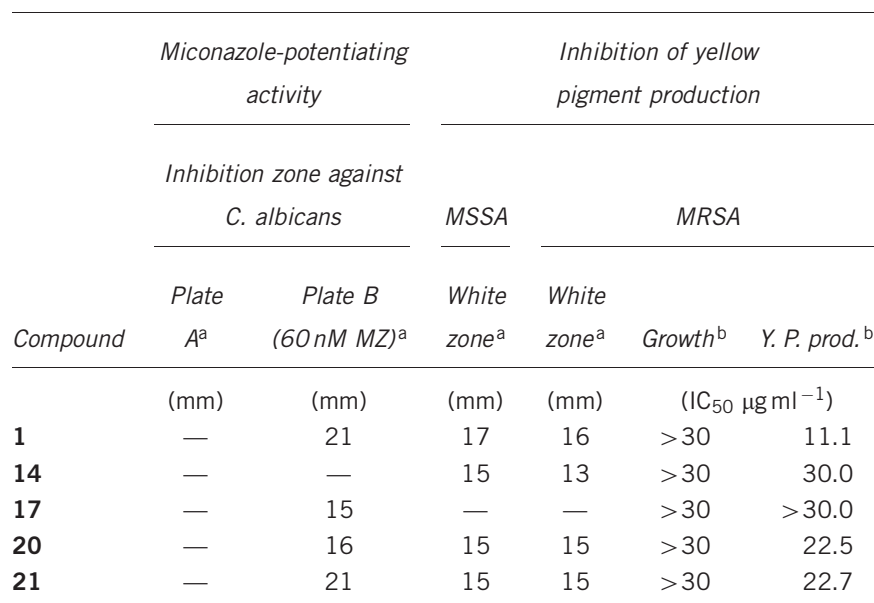

Abbreviations: -, No inhibition zone or no white zone; MRSA, methicillin-resistant Staphylococcus aureus; MZ, miconazole; Y. P. prod., yellow pigment production. apaper disk method $(20 \mu \mathrm{g}$ per sample per $6 \mathrm{~mm}$ disk).

bLiquid culture method.

$\mathrm{Hz}, 1 \mathrm{H}), 1.73(\mathrm{~s}, 3 \mathrm{H}), 1.65(\mathrm{~s}, 3 \mathrm{H}), 1.30(\mathrm{~d}, J=7.9 \mathrm{~Hz}, 3 \mathrm{H}) .{ }^{13} \mathrm{C}-\mathrm{NMR}$ $\left(75 \mathrm{MHz}, \mathrm{CDCl}_{3}\right) \delta: 164.9,163.1,150.5,133.9,133.6,128.5,127.6,127.2$, $126.3,113.4,111.1,103.8,56.7,49.0,26.3,20.3,14.8$. high resolution mass spectrum (HRMS) (ESI) $[\mathrm{M}+\mathrm{Na}]^{+}$calcd for $\mathrm{C}_{19} \mathrm{H}_{19} \mathrm{NNaO}_{2}=316.1314$, found $=316.1331$.

4-Hydroxy-3-((1R,4S,5R)-4-(hydroxymethyl)-2,5-dimethylcyclopent-2-en-1-yl)-5phenylpyridin-2(1H)-one (7). $[\alpha]_{\mathrm{D}}^{27}+39.2\left(\right.$ c $\left.0.5, \mathrm{CHCl}_{3}\right)$. IR $(\mathrm{KBr}) \mathrm{cm}^{-1}$ : $3373,2925,2361,1639,1433,1214 .{ }^{1} \mathrm{H}-\mathrm{NMR}\left(400 \mathrm{MHz}, \mathrm{CDCl}_{3}\right) \delta: 7.54-7.34$ $(\mathrm{m}, 6 \mathrm{H}), 5.53(\mathrm{~s}, 1 \mathrm{H}), 4.04(\mathrm{bd}, J=6.8 \mathrm{~Hz}, 1 \mathrm{H}), 3.87(\mathrm{dd}, J=12.0,2.2 \mathrm{~Hz}$, $1 \mathrm{H}), 3.67$ (dd, $J=12.0,2.2 \mathrm{~Hz}, 1 \mathrm{H}), 2.61-2.57(\mathrm{~m}, 1 \mathrm{H}), 2.35-2.29(\mathrm{~m}, 1 \mathrm{H})$, $1.64(\mathrm{~s}, 3 \mathrm{H}), 1.28(\mathrm{~d}, J=6.8 \mathrm{~Hz}, 3 \mathrm{H}) .{ }^{13} \mathrm{C}-\mathrm{NMR}\left(150 \mathrm{MHz}, \mathrm{CDCl}_{3}\right) \delta: 166.7$, $160.8,140.2,132.9,131.6,129.8,129.3,128.8,128.6,122.5,112.3,63.8$,
55.2, 51.9, 41.2, 20.9, 15.1. HRMS (ESI) $[\mathrm{M}+\mathrm{Na}]^{+}$calcd for $\mathrm{C}_{19} \mathrm{H}_{21} \mathrm{NNaO}_{3}=334.1521$, found $=334.1518$.

(5aR,6R,7R,8R,8aS)-7-(Hydroxymethyl)-6-iodo-5a,8-dimethyl-4-phenyl-2,5a,6,7, 8,8a-hexahydro-1H-cyclopenta[4,5]furo[3,2-c]pyridin-1-one $(11) . \quad[\alpha]_{\mathrm{D}}^{20}-56.0$ (c 1.0, $\left.\mathrm{CH}_{3} \mathrm{OH}\right)$. IR (KBr) cm ${ }^{-1}: 3321,2960,2872,1656,1429,1379,1033$. ${ }^{1} \mathrm{H}-\mathrm{NMR}\left(400 \mathrm{MHz}, \mathrm{CD}_{3} \mathrm{OD}\right) \delta: 7.53-7.50(\mathrm{~m}, 2 \mathrm{H}), 7.41(1 \mathrm{H}), 7.40-7.36$ $(\mathrm{m}, 2 \mathrm{H}), 7.33-7.28(\mathrm{~m}, 1 \mathrm{H}), 4.47(\mathrm{~d}, J=14.3 \mathrm{~Hz}, 1 \mathrm{H}), 3.76(\mathrm{dd}, J=11.8$, $2.6 \mathrm{~Hz}, 1 \mathrm{H}), 3.72(\mathrm{dd}, J=11.8,2.3 \mathrm{~Hz}, 1 \mathrm{H}), 3.14(\mathrm{~d}, J=9.8 \mathrm{~Hz}, 1 \mathrm{H}), 2.10-2.00$ $(\mathrm{m}, 1 \mathrm{H}), 1.73-1.63(\mathrm{~m}, 1 \mathrm{H}), 1.67(\mathrm{~s}, 3 \mathrm{H}), 1.40(\mathrm{~d}, J=6.7 \mathrm{~Hz}, 3 \mathrm{H}) .{ }^{13} \mathrm{C}-\mathrm{NMR}$ $\left(100 \mathrm{MHz}, \mathrm{CD}_{3} \mathrm{OD}\right) \delta: 166.1,163.3,135.6,134.2,129.7,128.7,128.5,114.6$, $112.9,99.0,58.8,57.0,56.5,41.6,39.9,29.6,19.6$. HRMS (ESI) $[\mathrm{M}+\mathrm{H}]^{+}$ calcd for $\mathrm{C}_{19} \mathrm{H}_{21} \mathrm{INO}_{3}=438.0566$, found $=438.0551$.

(5aS,8S,8aS)-5a,8-Dimethyl-4-phenyl-2,5a,8,8a-tetrahydro-1H-cyclopenta[4,5] furo[3,2-c]pyridin-1-one (14). $[\alpha]_{\mathrm{D}}^{27}-129.8\left(\right.$ c $\left.1.0, \mathrm{CHCl}_{3}\right) . \mathrm{IR}(\mathrm{KBr}) \mathrm{cm}^{-1}$ : 2926, 1739, 1655, 1501, 1459, 1428, 1369, 1263, 1227, 1150, 1096. ${ }^{1} \mathrm{H}-\mathrm{NMR}$ $\left(300 \mathrm{MHz}, \mathrm{CDCl}_{3}\right) \delta: 7.54-7.50(\mathrm{~m}, 3 \mathrm{H}), 7.43-7.36(\mathrm{~m}, 1 \mathrm{H}), 7.34-7.28$ $(\mathrm{m}, 2 \mathrm{H}), 5.95(\mathrm{dd}, J=5.6,2.1 \mathrm{~Hz}, 1 \mathrm{H}), 5.73(\mathrm{dd}, J=5.4,1.5 \mathrm{~Hz}, 1 \mathrm{H}), 3.25$ (s, $1 \mathrm{H}), 3.14-3.12(\mathrm{~m}, 1 \mathrm{H}), 1.69(\mathrm{~s}, 3 \mathrm{H}), 1.29(\mathrm{~d}, J=7.5 \mathrm{~Hz}, 3 \mathrm{H}) .{ }^{13} \mathrm{C}-\mathrm{NMR}$ $\left(75.5 \mathrm{MHz}, \mathrm{CDCl}_{3}\right) \delta: 141.4,134.5,133.2,131.1,128.5,127.6,127.4,127.4$, 104.8, 55.5, 46.3, 29.5, 26.0, 21.6. HRMS (ESI) $[\mathrm{M}+\mathrm{Na}]^{+}$calcd for $\mathrm{C}_{18} \mathrm{H}_{17} \mathrm{NNaO}_{2}=302.1157$, found $=302.1142$.

(5aS,8S,8aS)-4-(4-((2-Methoxyethoxy)methoxy)phenyl)-5a,8-dimethyl-2,5a,8,8atetrahydro-1H-cyclopenta[4,5] furo[3,2-c]pyridin-1-one $\quad(15) .[\alpha]_{\mathrm{D}}^{25}-59.2$ (c 1.0, $\left.\mathrm{CHCl}_{3}\right)$. IR ( $\left.\mathrm{KBr}\right) \mathrm{cm}^{-1}: 3401,2925,1652,1614,1514,1445,1228$, $1103,1000,833 .{ }^{1} \mathrm{H}-\mathrm{NMR}\left(400 \mathrm{MHz}, \mathrm{CDCl}_{3}\right) \delta: 7.58-7.56(\mathrm{~m}, 1 \mathrm{H}), 7.45-7.41$ $(\mathrm{m}, 2 \mathrm{H}), 7.09-7.06(\mathrm{~m}, 2 \mathrm{H}), 5.94(\mathrm{dd}, J=7.6,1.8 \mathrm{~Hz}, 1 \mathrm{H}), 5.72(\mathrm{dd}, J=7.6$, $1.8 \mathrm{~Hz}, 1 \mathrm{H}), 5.29(\mathrm{~s}, 2 \mathrm{H}), 3.85-3.82(\mathrm{~m}, 2 \mathrm{H}), 3.58-3.55(\mathrm{~m}, 2 \mathrm{H}), 3.38(\mathrm{~s}, 3 \mathrm{H})$, $3.24(\mathrm{~s}, 1 \mathrm{H}), 3.13-3.08(\mathrm{~m}, 1 \mathrm{H}), 1.69(\mathrm{~s}, 3 \mathrm{H}), 1.27(\mathrm{~d}, J=6.8 \mathrm{~Hz}, 3 \mathrm{H})$. ${ }^{13} \mathrm{C}-\mathrm{NMR}\left(150 \mathrm{MHz}, \mathrm{CDCl}_{3}\right) \delta: 165.5,161.2,156.7,141.3,133.9,131.0,128.8$, $126.5,116.3,104.9,93.4,71.6,67.6,59.0,55.4,46.4,26.2,21.5$. HRMS (ESI) $[\mathrm{M}+\mathrm{H}]^{+}$calcd for $\mathrm{C}_{22} \mathrm{H}_{26} \mathrm{NO}_{5}=384.1811$, found $=384.1824$.

(5aS,8S,8aS)-4-(4-Hydroxyphenyl)-5a,8-dimethyl-2,5a,8,8a-tetrahydro-1H-cyclopenta[4,5]furo[3,2-c]pyridin-1-one (16). [ $\alpha]_{\mathrm{D}}^{25}-9.91\left(c \quad 0.5, \mathrm{CH}_{3} \mathrm{OH}\right) . \quad \mathrm{IR}$ $(\mathrm{KBr}) \mathrm{cm}^{-1}: 3435,1646,1516,1430,1270,1108,1042,834,786,630$. ${ }^{1} \mathrm{H}-\mathrm{NMR}\left(400 \mathrm{MHz}, \mathrm{CD}_{3} \mathrm{OD}\right) \delta: 7.35-7.30(\mathrm{~m}, 3 \mathrm{H}), 6.81-6.77(\mathrm{~m}, 2 \mathrm{H})$, 
$5.98(\mathrm{dd}, J=7.6,2.4 \mathrm{~Hz}, 1 \mathrm{H}), 5.73(\mathrm{dd}, J=7.6,1.8 \mathrm{~Hz}, 1 \mathrm{H}), 3.18(\mathrm{~d}, J=1.6 \mathrm{~Hz}$, $1 \mathrm{H}), \quad 3.04-2.97(\mathrm{~m}, 1 \mathrm{H}), 1.67(\mathrm{~s}, 3 \mathrm{H}), 1.25(\mathrm{~d}, J=7.2 \mathrm{~Hz}) .{ }^{13} \mathrm{C}-\mathrm{NMR}$ $\left(100 \mathrm{MHz}, \mathrm{CD}_{3} \mathrm{OD}\right) \delta: 166.8,163.0,158.0,133.9,132.0,129.7,125.3,116.1$, $114.5,113.0,105.4,56.7,26.2$, 21.6. HRMS (ESI) $[\mathrm{M}+\mathrm{Na}]^{+}$calcd for $\mathrm{C}_{18} \mathrm{H}_{17} \mathrm{NNaO}_{3}=318.1106$, found $=318.1108$.

(5aS,8R,8aS)-5a,8-Dimethyl-1-oxo-4-phenyl-2,5a,8,8a-tetrahydro-1H-cyclopenta $[4,5]$ furo[3,2-c]pyridine-7-carbaldehyde (17). $[\alpha]_{\mathrm{D}}^{20}-191.0$ (c 1.0, $\left.\mathrm{CHCl}_{3}\right)$. IR $(\mathrm{KBr}) \mathrm{cm}^{-1}: 2969,1689,1652,1456,1430,1373,1227 .{ }^{1} \mathrm{H}-\mathrm{NMR}(400 \mathrm{MHz}$, $\left.\mathrm{CDCl}_{3}\right) \delta: 9.83(\mathrm{~s}, 1 \mathrm{H}), 7.53-7.30(\mathrm{~m}, 6 \mathrm{H}), 6.58(\mathrm{~s}, 1 \mathrm{H}), 3.48(\mathrm{q}, J=7.2 \mathrm{~Hz}$, $1 \mathrm{H}), 3.41(\mathrm{~s}, 1 \mathrm{H}), 1.80(\mathrm{~s}, 3 \mathrm{H}), 1.38(\mathrm{~d}, J=7.1 \mathrm{~Hz}, 3 \mathrm{H}) .{ }^{13} \mathrm{C}-\mathrm{NMR}(100 \mathrm{MHz}$, $\left.\mathrm{CDCl}_{3}\right) \delta: 190.0,164.3,162.9,151.8,147.2,134.7,133.0,128.5,127.5,127.3$, $112.3,110.8,101.3,56.8,42.4,25.1,20.4$. HRMS (ESI) $[\mathrm{M}+\mathrm{Na}]^{+}$calcd for $\mathrm{C}_{19} \mathrm{H}_{17} \mathrm{NNaO}_{3}=30.1106$, found $=330.1077$.

(5aS,8R,8aS)-7-(Hydroxymethyl)-5a,8-dimethyl-4-phenyl-2,5a,8,8a-tetrahydro-1H-

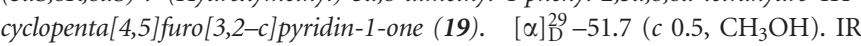
$(\mathrm{KBr}) \mathrm{cm}^{-1}: 3460,2849,2150,1650,1453,1430,1112 .{ }^{1} \mathrm{H}-\mathrm{NMR}(400 \mathrm{MHz}$, $\left.\mathrm{CD}_{3} \mathrm{OD}\right) \delta: 7.50(\mathrm{~d}, J=8.4 \mathrm{~Hz}, 2 \mathrm{H}), 7.44(\mathrm{~s}, 1 \mathrm{H}), 7.37(\mathrm{t}, J=8.4 \mathrm{~Hz}, 2 \mathrm{H})$, $7.31-7.27(\mathrm{~m}, 1 \mathrm{H}), 5.65(\mathrm{~s}, 1 \mathrm{H}), 4.18(\mathrm{~d}, J=15.6 \mathrm{~Hz}, 1 \mathrm{H}), 4.10(\mathrm{~d}, J=15.6 \mathrm{~Hz}$, $1 \mathrm{H}), 3.28(\mathrm{~d}, J=0.8 \mathrm{~Hz}, 1 \mathrm{H}), 3.01(\mathrm{q}, J=7.2 \mathrm{~Hz}, 1 \mathrm{H}), 1.68(\mathrm{~s}, 3 \mathrm{H}), 1.30$ $(\mathrm{d}, J=7.2 \mathrm{~Hz}, 3 \mathrm{H}) .{ }^{13} \mathrm{C}-\mathrm{NMR}\left(150 \mathrm{MHz}, \mathrm{CD}_{3} \mathrm{OD}\right) \delta: 166.5,163.3,155.5$, $135.1,129.5,128.7,128.5,128.4,126.3,114.8,113.0,104.7,60.2,58.0,47.0$, 26.3, 20.8. HRMS (ESI) $[\mathrm{M}+\mathrm{Na}]^{+}$calcd for $\mathrm{C}_{19} \mathrm{H}_{19} \mathrm{NNaO}_{3}=332.1262$, found $=332.1276$

(4bS,5R,7aS)-5,6,7a-Trimethyl-3-phenyl-4b,5-dihydro-1H-cyclopenta[4,5]furo[2,

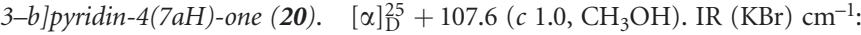
$2965,1653,1454,1432,1217,1041 .{ }^{1} \mathrm{H}-\mathrm{NMR}\left(400 \mathrm{MHz}, \mathrm{CDCl}_{3}\right) \delta: 7.54-7.51$ $(\mathrm{m}, 3 \mathrm{H}), 7.41-7.37(\mathrm{~m}, 2 \mathrm{H}), 7.32-7.28(\mathrm{~m}, 1 \mathrm{H}), 5.40(\mathrm{bs}, 1 \mathrm{H}), 3.28(\mathrm{~d}$, $J=1.0 \mathrm{~Hz}, 1 \mathrm{H}), 2.90(\mathrm{bq}, J=7.0 \mathrm{~Hz}, 1 \mathrm{H}), 1.73(\mathrm{~s}, 3 \mathrm{H}), 1.67(\mathrm{~s}, 3 \mathrm{H}), 1.30$ $(\mathrm{d}, \quad J=7.0 \mathrm{~Hz}, \quad 3 \mathrm{H}) .{ }^{13} \mathrm{C}-\mathrm{NMR} \quad\left(100 \mathrm{MHz}, \quad \mathrm{CDCl}_{3}\right) \quad \delta: \quad 165.4, \quad 162.7$, $150.7,134.3,133.5,128.7,127.7,127.4,126.4,111.8,104.3,56.6,49.2,26.4$, 20.4, 14.9. HRMS (ESI) $[\mathrm{M}+\mathrm{H}]^{+}$calcd for $\mathrm{C}_{19} \mathrm{H}_{20} \mathrm{NO}_{2}=294.1494$, found $=294.1485$.

(5aR,8S,8aR)-5a,7,8-Trimethyl-4-phenyl-2,5a,8,8a-tetrahydro-1H-cyclopenta[4,5] furo[3,2-c]pyridin-1-one (ent-Citridone A, 21). $[\alpha]_{\mathrm{D}}^{23}+77.6\left(c 0.1, \mathrm{CH}_{3} \mathrm{OH}\right)$. IR $(\mathrm{KBr}) \mathrm{cm}^{-1}: 2924,1652,1431,1204,1034,765,697 .{ }^{1} \mathrm{H}-\mathrm{NMR}(400 \mathrm{MHz}$, $\left.\mathrm{CDCl}_{3}\right) \delta: 7.54-7.51(\mathrm{~m}, 2 \mathrm{H}), 7.47-7.34(\mathrm{~m}, 4 \mathrm{H}), 5.43(\mathrm{dq}, J=1.4 \mathrm{~Hz}, 1 \mathrm{H})$, 3.33 (bs, $1 \mathrm{H}), 2.88(\mathrm{bq}, J=7.0 \mathrm{~Hz}, 1 \mathrm{H}), 1.74(\mathrm{~s}, 3 \mathrm{H}), 1.70(\mathrm{~s}, 3 \mathrm{H}), 1.30$ $(\mathrm{d}, J=7.2 \mathrm{~Hz}, 3 \mathrm{H}) .{ }^{13} \mathrm{C}-\mathrm{NMR}\left(100 \mathrm{MHz}, \mathrm{CDCl}_{3}\right) \delta: 164.1,163.1,150.7,133.7$, $133.4,128.7,127.8,127.5,126.4,113.1,111.9,104.2,56.6,49.2,26.4$, 20.4, 14.9. HRMS (ESI) $[\mathrm{M}+\mathrm{H}]^{+}$calcd for $\mathrm{C}_{19} \mathrm{H}_{20} \mathrm{NO}_{2}=294.1494$, found $=294.1488$

(5aS $\left.{ }^{*}, 8 a S^{*}\right)$-4-Phenyl-2,5a,8,8a-tetrahydro-1H-cyclopenta[4,5]furo[3,2-c]pyridin1-one (27). IR ( $\mathrm{KBr}) \mathrm{cm}^{-1}: 3055,2929,2360,2340,1649,1598,1230,1062$, 1197, 762. ${ }^{1} \mathrm{H}-\mathrm{NMR}\left(400 \mathrm{MHz}, \mathrm{CDCl}_{3}\right) \quad \delta: 7.65-7.61(\mathrm{~m}, 1 \mathrm{H}), 7.52-7.50$ $(\mathrm{m}, 2 \mathrm{H}), 7.42-7.38(\mathrm{~m}, 2 \mathrm{H}), 7.34-7.30(\mathrm{~m}, 1 \mathrm{H}), 6.15(\mathrm{~d}, J=5.2 \mathrm{~Hz}, 1 \mathrm{H}), 6.05$ $(\mathrm{d}, J=5.2 \mathrm{~Hz}, 1 \mathrm{H}), 5.89(\mathrm{dd}, J=5.2,2.4 \mathrm{~Hz}, 1 \mathrm{H}), 4,15(\mathrm{t}, J=7.8 \mathrm{~Hz}, 1 \mathrm{H}), 2.90$ $(\mathrm{d}, J=7.8 \mathrm{~Hz}, 1 \mathrm{H}), 2.85(\mathrm{t}, J=2.4 \mathrm{~Hz}, 1 \mathrm{H}) .{ }^{13} \mathrm{C}-\mathrm{NMR}\left(150 \mathrm{MHz}, \mathrm{CDCl}_{3}\right)$ $\delta: 166.2,162.1,137.4,134.5,132.9,128.6,128.0,127.6,127.6,113.3,112.2$, 95.7, 40.9, 38.2. HRMS (ESI) $[\mathrm{M}+\mathrm{Na}]^{+}$calcd for $\mathrm{C}_{16} \mathrm{H}_{13} \mathrm{NNaO}_{2}=274.0844$, found $=274.0841$

$\left(4 b S^{*}, 7 a S^{*}\right)$-3-Phenyl-4b,5-dihydro-1H-cyclopenta[4,5]furo[2,3-b]pyridin-4(7aH)one (29). IR (KBr) cm${ }^{-1}$ : 3020, 2929, 2857, 2400, 1642, 1597, 1477, 1216. ${ }^{1} \mathrm{H}-\mathrm{NMR}\left(400 \mathrm{MHz}, \mathrm{CD}_{3} \mathrm{OD}\right) \delta: 7.48-7.45(\mathrm{~m}, 3 \mathrm{H}), 7.39-7.35(\mathrm{~m}, 2 \mathrm{H})$, $7.32-7.28(\mathrm{~m}, 1 \mathrm{H}), 6.20-6.17(\mathrm{~m}, 1 \mathrm{H}), 5.98(\mathrm{~d}, J=8.0 \mathrm{~Hz}, 1 \mathrm{H}), 5.92-5.89$ $(\mathrm{m}, 1 \mathrm{H}), 4.14(\mathrm{td}, J=8.0,2.4 \mathrm{~Hz}, 1 \mathrm{H}), 2.89(\mathrm{ddt}, J=17.6,8.0,2.4 \mathrm{~Hz}, 1 \mathrm{H})$, $2.69(\mathrm{dt}, J=17.6,2.4 \mathrm{~Hz}, 1 \mathrm{H}) .{ }^{13} \mathrm{C}-\mathrm{NMR}\left(150 \mathrm{MHz}, \mathrm{CD}_{3} \mathrm{OD}\right) \delta: 169.4,163.9$, $138.8,136.5,130.4,129.2,129.0,128.2,111.1,95.6,41.8,39.7$. HRMS (ESI) $[\mathrm{M}+\mathrm{H}]^{+}$calcd for $\mathrm{C}_{16} \mathrm{H}_{14} \mathrm{NO}_{2}=252.1024$, found $=252.1012$.
Assay for miconazole-potentiating activity against $C$. albicans using paper disks

C. albicans ATCC 64548 was inoculated into a 50-ml test tube containing $10 \mathrm{ml}$ seed medium (potato extract containing peptone $0.50 \%$ and glucose $1.0 \%$ ), and was grown for $24 \mathrm{~h}$ on a rotary shaker. In Method A, the seed culture of C. albicans $(0.10 \%, \mathrm{v} / \mathrm{v})$ was transferred to two different agar plates, GY agar (glucose $1.0 \%$, yeast extract $0.50 \%$ and agar $0.80 \%$; plate A) and GY agar plus miconazole $(60 \mathrm{~nm}$; plateB). The concentration $(60 \mathrm{~nm})$ of miconazole is onefourth of the MIC value against C. albicans, and showed no effect on the growth of C. albicans. Paper disks (6 mm; ADVANTEC, Tokyo, Japan) containing a $20-\mu \mathrm{g}$ sample were placed on plates $\mathrm{A}$ and $\mathrm{B}$, which were incubated at $27^{\circ} \mathrm{C}$ for $24 \mathrm{~h}$. Samples showing inhibition zones selectively on plate $\mathrm{B}$ were selected as potentiators of miconazole activity against $C$. albicans.

\section{Assay for inhibition of yellow pigment production in MRSA using paper disks}

MRSA K-24 strain, a clinical isolate, was used as a yellow pigment-producing strain. MRSA was cultured in Mueller-Hinton broth at $37^{\circ} \mathrm{C}$ for $20 \mathrm{~h}$ and adjusted to $1 \times 10^{8} \mathrm{CFU}$ per ml. The inoculum $(100 \mu \mathrm{l})$ was spread on $25 \mathrm{ml}$ TYB agar (tryptone $1.7 \%$, yeast extract $1.0 \%, \mathrm{NaCl} 0.5 \%, \mathrm{~K}_{2} \mathrm{HPO}_{4} 0.25 \%$, agar $1.5 \%$ and glycerol monoacetate $1.5 \%)$ on a plate $(100 \times 140 \mathrm{~mm})$. Paper disks ( $6 \mathrm{~mm}$ i.d.) containing a $20-\mu \mathrm{g}$ sample were placed on the plate and incubated at $37^{\circ} \mathrm{C}$ for $72 \mathrm{~h}$. Inhibition of the production of yellow pigments by a sample is expressed as the diameter $(\mathrm{mm})$ of the white zone on the plate.

\section{Assay for growth and yellow pigment production in MRSA by liquid culture}

A mixture containing TYB $(980 \mu \mathrm{l})$, a sample $(10 \mu \mathrm{l})$ and MRSA $(10 \mu \mathrm{l}$, at a final concentration of $1 \times 10^{7} \mathrm{CFU}$ per $\mathrm{ml}$ ) in a total volume of $1000 \mu \mathrm{l}$ was incubated on a rotary shaker at 210 r.p.m. for $72 \mathrm{~h}$ at $37^{\circ} \mathrm{C}$. (1) MRSA growth: the culture's turbidity was determined at $600 \mathrm{~nm}$ using a Power Wave x 340 (BIO-TEK Instruments Inc., Winooski, VT, USA). (2) Yellow pigment production: after the culture was centrifuged, yellow pigments in MRSA mycelia were extracted with methanol $(500 \mu \mathrm{l})$ at $60^{\circ} \mathrm{C}$ for $2 \mathrm{~h}$ in the dark. The absorbance of yellow pigments was determined at $450 \mathrm{~nm}$ using a Power Wave $\times 340$. Inhibition of MRSA growth and yellow pigment production by a sample (\% of control) is defined as (absorbance-sample/absorbancecontrol $) \times 100$. The $\mathrm{IC}_{50}$ values are defined as the sample concentrations that cause 50\% inhibition of MRSA growth and yellow pigment production.

\section{ACKNOWLEDGEMENTS}

We thank Ms Noriko Sato and Dr Kenichiro Nagai (School of Pharmaceutical Sciences, Kitasato University) for measuring NMR spectra and MS data. We thank Ms Minori Shinkai and Ms Eri Sasaki for measuring the activity of citridones. This work was supported by Takeda Science Foundation and JSPS KAKENHI Grant Number 25870704

1 Fukuda, T., Yamaguchi, Y., Masuma, R., Tomoda, H. \& Ōmura, S. Citridones, new potentiators of antifungal miconazole activity, produced by Penicillium sp. FKI-1938 I. Taxonomy, fermentation, isolation and biological properties. J. Antibiot. 58, 309-314 (2005).

2 Fukuda, T., Tomoda, H. \& Ōmura, S. Citridones, new potentiators of antifungal miconazole activity, produced by Penicillium sp. FKI-1938 II. Structure elucidation. J. Antibiot. 58, 315-321 (2005).

3 Fukuda, T., Sakabe, Y., Tomoda, H. \& Ōmura, S. Fungal citridone D having a novel phenylfuropyridine skeleton. Chem. Pharm. Bull. 54, 1659-1661 (2006).

4 Miyagawa, T. et al. Total synthesis of citridone A. Org. Lett. 13, 1158-1161 (2011).

5 Fotiadou, A.-D. \& Zografos, A.-L. Accessing the structural diversity of pyridone alkaloids: concise total synthesis of rac-citridone A. Org. Lett. 13, 4592-4595 (2011).

6 Genaux, C.-T. \& Walters, W.-D. The thermal decomposition of cyclobutane. J. Am Chem. Soc. 73, 4497-4498 (1951).

7 Kern, F. \& Walters, W.-D. The thermal decomposition of cyclobutane. Proc. Natl Acad. Sci. USA 38, 937-942 (1952).

8 Genaux, C.-T., Kern, F. \& Walters, W.-D. The thermal decomposition of cyclobutane. J. Am. Chem. Soc. 75, 6196-6199 (1953) 
9 Sakai, K. et al. Method of search for microbial inhibitors of staphyloxanthin production by MRSA. Biol. Pharm. Bull. 35, 48-53 (2012).

10 Marshall, J.-H. \& Wilmoth, G.-J. Pigments of Staphylococcus aureus, a series of triterpenoid carotenoids. J. Bacteriol. 147, 900-913 (1981).

11 Marshall, J.-H. \& Wilmoth, G.-J. Proposed pathway of triterpenoid carotenoid biosynthesis in Staphylococcus aureus: evidence from a study of mutants. J. Bacteriol 147, 914-919 (1981).

12 Wang, R. et al. Identification of novel cytolytic peptides as key virulence determinants for community-associated MRSA. Nat. Med. 13, 1510-1514 (2007).

13 Mitchell, G. et al. Tomatidine acts in synergy with aminoglycoside antibiotics against multiresistant Staphylococcus aureus and prevents virulence gene expression. J. Antimicrob. Chemother. 67, 559-568 (2012).

14 Lang, S., Livesley, M.-A., Lambert, P.-A., Littler, W.-A. \& Elliott, T.-S. Identification of a novel antigen from Staphylococcus epidermidis. FEMS. Immunol. Med. Microbio/ 29, 213-220 (2000).

15 Clauditz, A., Resch, A., Wieland, K.-P., Peschel, A. \& Goötz, F. Staphyloxanthin plays a role in the fitness of Staphylococcus aureus and its ability to cope with oxidative stress. Infect. Immun 74, 4950-4953 (2006).
16 Mishra, N.-N. et al. Carotenoid-related alteration of cell membrane fluidity impacts Staphylococcus aureus susceptibility to host defense peptides. Antimicrob. Agents Chemother 55, 526-531 (2011).

17 Liu, G.-Y. et al. Staphylococcus aureus golden pigment impairs neutrophil killing and promotes virulence through its antioxidant activity. J. Exp. Med. 202, 209-215 (2005).

18 Pelz, A. et al. Structure and biosynthesis of staphyloxanthin from Staphylococcus aureus. J. Biol. Chem. 280, 32493-32498 (2005).

19 Liu, C.-I. et al. A cholesterol biosynthesis inhibitor blocks Staphylococcus aureus virulence. Science 319, 1391-1394 (2008).

20 Liu, C.-I., Jeng, W.-Y., Chang, W.-J., Ko, T.-P. \& Wang, A.-H. Binding modes of zaragozic acid $A$ to human squalene synthase and staphylococcal dehydrosqualene synthase. J. Biol. Chem. 287, 18750-18757 (2012).

21 Leejae, S., Hasap, L. \& Voravuthikunchai, S.-P. Inhibition of staphyloxanthin biosynthesis in Staphylococcus aureus by rhodomyrtone, a novel antibiotic candidate. J. Med. Microbiol. 62, 421-428 (2013).

22 Fukuda, T., Nagai, K. \& Tomoda, H. ( \pm )-Tylopilusins, diphenolic metabolites from the fruiting bodies of Tylopilus eximius. J. Nat. Prod. 75, 2228-2231 (2012).

Supplementary Information accompanies the paper on The Journal of Antibiotics website (http://www.nature.com/ja) 